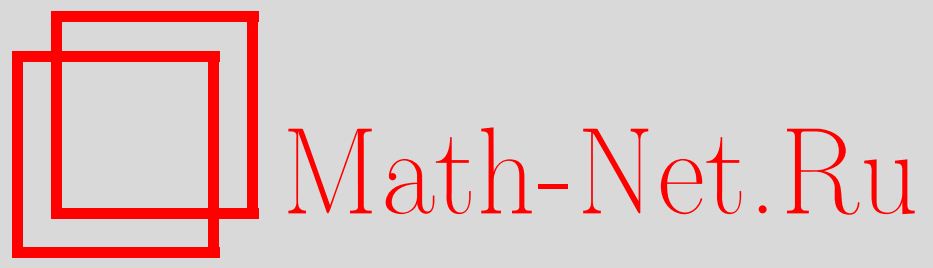

Обшероссийский математический портал

А. В. Сильниченко, О скорости сходимости жадных алгоритмов, Матем. заметки, 2004, том 76, выпуск 4, 628-632

DOI: https://doi.org/10.4213/mzm579

Использование Общероссийского математического портала Math-Net.Ru подразумевает, что вы прочитали и согласны с пользовательским соглашением http://www. mathnet.ru/rus/agreement

Параметры загрузки:

IP : 35.173 .219 .12

26 апреля 2023 г., 18:27:52 


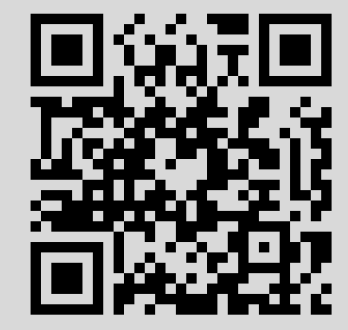




\section{О СКОРОСТИ СХОДИМОСТИ ЖАДНЫХ АЛГОРИТМОВ}

\section{А. В. Сильниченко}

Введение. В данной работе речь пойдет о приближении элементов вещественного сепарабельного гильбертова пространства $H$ со скалярным произведением $(\cdot, \cdot)$. Подмножество элементов $D \subset H$ назовем словарем, если $\|g\|=1$ для любого $g \in D$ и при этом замькание линейной оболочки $D$ в $H$ совпадает с $H$. Для $f \in H$ обозначим через $g=g(f) \in D$ элемент из $D$, который максимизирует $|(f, g)|$ (предполагаем, что такой элемент всегда существует). Определим

$$
G(f)=G(f, D)=(f, g) g, \quad R(f)=R(f, d)=f-G(f) .
$$

Определим чистый жадный алгоритм как алгоритм построения последовательностей $G_{m}(f)$ и $R_{m}(f)$ следующим образом: $R_{0}(f)=f, G_{0}(f)=0$, далее по индукции

$$
G_{m}(f)=G_{m-1}(f)+G\left(R_{m-1}(f)\right), \quad R_{m}(f)=f-G_{m}(f)=R\left(R_{m-1}(f)\right) .
$$

Определим для словаря $D$ класс элементов

$$
A_{1}^{0}(D, M)=\left\{f \in H: f=\sum_{k \in \Lambda} c_{k} w_{k}, \quad w_{k} \in D,|\Lambda|<\infty, \sum_{k \in \Lambda}\left|c_{k}\right| \leqslant M\right\} .
$$

Пусть $A_{1}(D, M)$ - замыкание в $H$ множества $A_{1}^{0}(D, M)$. Положим

$$
A_{1}(D)=\bigcup_{M>0} A_{1}(D, M)
$$

Для $f \in A_{1}(D)$ обозначим

$$
|f|_{A_{1}(D)}=\min _{f \in A_{1}(D, M)} M
$$

В данных обозначениях сформулируем проблему оценки скорости сходимости жадного алгоритма следуюшим образом: оценить величину

$$
f(m)=\sup \left\|f_{m}-G_{m}(f, D)\right\| \cdot|f|_{A_{1}(D)}^{-1}
$$

в зависимости от $m$.

Работа выполнена при финансовой поддержке программы "Ведущие научные школы", грант № НШ-304.2003.1. 
В 1996 г. Девором и Темляковым [1] получена оценка

$$
\left\|f-G_{m}(f)\right\| \leqslant m^{-1 / 6}|f|_{A_{1}(D)} ;
$$

в 1999 г. Конягин и Темляков [2] улучшили эту оценку:

$$
\left\|f-G_{m}(f)\right\| \leqslant 4 m^{-11 / 62}|f|_{A_{1}(D)} .
$$

С другой стороны, в 1996 г. Девором и Темляковьм [1] построен пример, когда

$$
\left\|f-G_{m}(f)\right\| \geqslant \frac{1}{2}|f|_{A_{1}(D)} m^{-1 / 2}, \quad m \geqslant 4
$$

Темляков и Лившиц [3] построили пример, когда

$$
\left\|f-G_{m}(f)\right\| \geqslant C|f|_{A_{1}(D)} m^{-0.27} .
$$

Здесь и далее через $C$ обозначим положительные абсолютные константы. В данной работе мы, развивая идеи [2], улучшаем оценку на скорость сходимости. При этом используются неравенства для коэффициентов жадных алгоритмов и строятся их непрерьвные аналоги (дифференциально-разностные неравенства для функций), после чего оценивается максимально возможный рост решений этих неравенств. Будет доказана

Теорема 1. Для любого словаря $D$ и любого әлемента $f \in A_{1}(D)$ верна оченка

$$
\left\|f-G_{m}(f, D)\right\| \leqslant C|f|_{A_{1}(D)} m^{-\gamma /(2(2+\gamma))}
$$

где $\gamma-$ корень уравнения $h(x)=0$,

$$
h(x)=(1+x)^{1 /(2+x)}\left(1+\frac{1}{1+x}\right)-1-\frac{1}{x},
$$

на отрезке $[1,1.5]$.

ЗАмечание 1 . Для сравнения $11 / 62=0.177 \ldots$, a $\gamma /(2(\gamma+2))=0.182 \ldots$ В качестве константы $C$ можно взять $C=1.7$.

ЗАмечание 2. Недавно Лившиц аннонсировал улучшение оценки снизу до $\left\|f-G_{m}(f)\right\| \geqslant C|f|_{A_{1}(D)} m^{-0.23}$ (устное сообщение). Таким образом, на данньй момент отношение между оценкой сверху и оценкой снизу меньше, чем $C m^{0.05}$.

Доказательство теоремы. Пусть

$$
\begin{array}{cl}
f_{m}=R_{m}(f), & a_{m}(f)=\left\|f_{m}\right\|^{2}, \quad d(f)=|(f, g(f))| \\
d_{m}=d\left(f_{m}\right), & b_{0}=|f|_{A_{1}(D)}, \quad b_{m+1}=b_{m}+d_{m}
\end{array}
$$

Будем использовать неравенства, доказанные в [1]:

$$
\begin{gathered}
a_{m} b_{m}^{-2} \leqslant m^{-1} \\
\left|f_{m}\right|_{A_{1}(D)} \leqslant b_{m}
\end{gathered}
$$

и неравенство, доказанное в [2]:

$$
|(u, v)| \leqslant d(u)|v|_{A_{1}(D)} .
$$

ЛЕмма 1. Уравнение $h(x)=0$ имеет единственный корень на отрезке $[1,1.5]$. 
ДоКАЗАТЕЛЬСтво. Легко проверить, что $h(1)<0, h(1.5)>0$ и $h^{\prime}(x)>0$ при $x \in[1,1.5]$, из чего следует утверждение леммы.

В силу (3) имеем

$$
\left|\left(f_{l}, f_{k}\right)\right| \leqslant d\left(f_{l}\right)\left\|f_{k}\right\|_{A_{1}(d)} \leqslant d_{l} b_{k}
$$

С другой стороны, при $l \geqslant k$ имеем

$$
\left|\left(f_{l}, f_{k}\right)\right|=\left|\left(f_{k}-\sum_{j=k}^{l-1}\left(f_{j}, g\left(f_{j}\right)\right) g\left(f_{j}\right), f_{k}\right)\right| \geqslant a_{k}-d_{k} \sum_{j=k}^{l-1} d_{j}=a_{k}-d_{k}\left(b_{l}-b_{k}\right) .
$$

Получили неравенство

$$
d_{l} \geqslant \frac{a_{k}-d_{k}\left(b_{l}-b_{k}\right)}{b_{k}} .
$$

Рассмотрим функции $A(t)$ и $B(t)$, где $t \geqslant 0$, определенные следующим образом: в точках $t=n$ положим $A(t)=a_{n}, B(t)=b_{n}$, и пусть $A$ и $B$ линейны на отрезках $[n, n+1]$. Тогда на отрезке $[n, n+1]$ имеем

$$
B^{\prime}(t)=d_{n}, \quad A^{\prime}(t)=-d_{n}^{2}=-\left(B^{\prime}(t)\right)^{2} .
$$

Сделаем замену переменных $x=B(t)$, и пусть $A=f(B)$; тогда $f_{x}^{\prime}(x)=-B_{t}^{\prime}(t)$, где в точках вида $x=B(n)$ рассматривается правая производная.

ЛЕмма 2. Функиия $f(t)$ удовлетворяет неравенству

$$
f^{\prime}\left(t_{2}\right) \leqslant \frac{-f\left(t_{1}\right)-f^{\prime}\left(t_{1}\right)\left(t_{2}-t_{1}\right)}{t_{1}}, \quad t_{1} \leqslant t_{2} .
$$

ДоКАЗАТЕльство. В случае $t_{1}=b_{k}, t_{2}=b_{l}$ неравенство следует из $(4)$. Пусть $t_{2} \in\left[b_{k}, b_{k+1}\right]$, $k \geqslant l$. Тогда

$$
b_{l} f^{\prime}\left(t_{2}\right) \leqslant-f\left(b_{l}\right)-f^{\prime}\left(b_{l}\right)\left(t_{2}-b_{l}\right)-f^{\prime}\left(b_{l}\right)\left(b_{k}-t_{2}\right) \leqslant-f\left(b_{l}\right)-f^{\prime}\left(b_{l}\right)\left(t_{2}-b_{l}\right),
$$

так как $f$ линейна на $\left[b_{k}, b_{k+1}\right]$ и $f^{\prime}\left(b_{l}\right)\left(b_{k}-t_{2}\right) \geqslant 0$.

Аналогично, для $t_{1} \in\left[b_{l}, b_{l+1}\right]$ имеем

$$
-f\left(b_{l}\right)-f^{\prime}\left(t_{1}\right)\left(t_{2}-b_{l}\right) \geqslant t_{1} f^{\prime}\left(t_{2}\right)+\left(b_{l}-t_{1}\right) f^{\prime}\left(t_{2}\right) \geqslant t_{1} f^{\prime}\left(t_{2}\right)
$$

так как $\left(b_{l}-t_{1}\right) f^{\prime}\left(t_{2}\right) \geqslant 0$. Далее,

$t_{1} f^{\prime}\left(t_{2}\right) \leqslant-f\left(t_{1}\right)+f\left(t_{1}\right)-f\left(b_{l}\right)-f^{\prime}\left(t_{1}\right)\left(t_{2}-t_{1}\right)+f^{\prime}\left(t_{1}\right)\left(b_{l}-t_{1}\right)=-f\left(t_{1}\right)-f^{\prime}\left(t_{1}\right)\left(t_{2}-t_{1}\right)$, так как $f\left(t_{1}\right)-f\left(b_{l}\right)=f^{\prime}\left(t_{1}\right)\left(t_{1}-b_{l}\right)$. Лемма доказана.

Итак мы получили неравенство (5). Найдем максимальное $\gamma$ такое, что функция $f(t)=t^{-\gamma}$ удовлетворяет этому неравенству. Подставив в (5), получим, что $\gamma-$ корень уравнения $h(x)=0$, причем при этом $\gamma$ неравенство обращается в равенство только при $t_{2}=t_{1} t_{0}$, где $t_{0}=$ $(1+\gamma)^{1 /(2+\gamma)}$. Сделаем замену переменных $t=e^{\tau}$. Пусть $g(\tau)=f\left(e^{\tau}\right) e^{\gamma \tau}$. Тогда (5) при $\tau_{1}=\tau_{2}$ примет вид

$$
g^{\prime}(\tau)+g(\tau) \leqslant 0
$$

а при $\tau_{2}-\tau_{1}=\tau_{0}$, где

получим

$$
\tau_{0}=\ln t_{0}=\frac{\ln (1+\gamma)}{2+\gamma}
$$

$$
U \tau_{0} g^{\prime}\left(\tau+\tau_{0}\right)+V \tau_{0} g^{\prime}(\tau) \leqslant g\left(\tau+\tau_{0}\right)-g(\tau)
$$

где

$$
U=\frac{1}{\gamma \tau_{0}}, \quad V=\frac{e^{\tau_{0}}-1}{\tau_{0} \gamma e^{-\tau_{0}-\gamma \tau_{0}}} .
$$


ПРЕДЛОЖЕНИЕ 1. ФунКиия $g(\tau)$ ограничена на $[0,+\infty]$.

ДоКАЗАТЕльСтво. Пусть $c_{1}=\max g(t)$ на отрезке $\left[0, \tau_{0}\right]$. Предположим, что существует $\tau \in\left[\tau_{0},+\infty\right]$ такое, что

$$
g(\tau)>\frac{U c_{1}+V c_{1}}{U-1}
$$

Учитывая, что $U>1, V>0$ и, стало быть, $\left(U c_{1}+V c_{1}\right) /(U-1)>0$, мы выводим существование такого $T>0$, что

$$
\max _{\tau \in\left[0, T+\tau_{0}\right]} g(\tau)=g\left(T+\tau_{0}\right) .
$$

Проинтегрируем неравенство (7) по отрезку $[0, T]$ и, обозначив $G(\tau)=\int_{0}^{x} g(\tau) d \tau$, получим

$$
U \tau_{0}\left(g\left(T+\tau_{0}\right)-g\left(\tau_{0}\right)\right)+V \tau_{0}(g(T)-g(0)) \leqslant G\left(T+\tau_{0}\right)-G\left(\tau_{0}\right)-G(T)
$$

или после преобразований

$$
0 \leqslant-U \tau_{0} g\left(T+\tau_{0}\right)+U \tau_{0} g\left(\tau_{0}\right)+V \tau_{0} g(0)+G\left(T+\tau_{0}\right)-G(T)-G\left(\tau_{0}\right) .
$$

Заметим, что $G\left(T+\tau_{0}\right)-G(T) \leqslant \tau_{0} g\left(T+\tau_{0}\right)$. Следовательно,

$$
0 \leqslant(1-U) \tau_{0} g\left(T+\tau_{0}\right)+U c_{1} \tau_{0}+V c_{1} \tau_{0}<\left(-U c_{1}-V c_{1}\right) \tau_{0}+U c_{1} \tau_{0}+V c_{1} \tau_{0}=0 .
$$

Получили противоречие. Тем самым, предложение доказано.

Из доказательства предложения 1 видно, что

$$
\max _{\tau \in[0,+\infty)} g(\tau) \leqslant C, \quad \text { где } C=c_{1} \frac{U c_{1}+V c_{1}}{U-1}, \quad c_{1}=\max _{\tau \in\left[0,+\tau_{0}\right]} g(\tau) .
$$

Оценим константу $c_{1}$. Из неравенства (6) вытекает, что

$$
\ln g(\tau) \leqslant \tau(\gamma-1)+\ln g(0)
$$

Учитывая условие $g(0) \in[0,1]$, мы получаем

$$
g(\tau) \leqslant g(0) e^{\tau(\gamma-1)} \leqslant e^{\tau(\gamma-1)} .
$$

Итак, $c_{1} \leqslant e^{\tau_{0}(\gamma-1)}$.

Вернувшись к функции $f$, получим неравенство

$$
f(t) \leqslant \frac{e^{\tau_{0}(\gamma-1)}(U+V)}{U-1} t^{-\gamma}
$$

В точках вида $b_{k}$ имеем

$$
a_{k} b_{k}^{\gamma} \leqslant \frac{e^{\tau_{0}(\gamma-1)}(U+V)}{U-1}
$$

Воспользовавшись неравенством (3), получим

$$
a_{k} \leqslant\left(\frac{e^{\tau_{0}(\gamma-1)}(U+V)}{U-1}\right)^{2 /(2+\gamma)} m^{-\gamma /(\gamma+2)}
$$

Подставив значения $\tau_{0}, \gamma, U, V$, легко убедиться, что $C<1.7$. Вспомнив, что $a_{k}=\left\|f_{k}\right\|^{2}$, мы завершаем доказательство теоремы 1 .

В [4] рассматривается следующее обобщение чистого жадного алгоритма. Фиксируется число $\beta \in(0,1]$. Элементы $G_{m}(f)$ и $R_{m}(f)$ определяются как

$$
G_{m}(f)=G_{m-1}(f)+\beta G\left(R_{m-1}(f)\right), \quad R_{m}(f)=f-G_{m}(f)=R\left(R_{m-1}(f)\right) .
$$


При $\beta=1$ мы получаем чистый жадный алгоритм.

Теорему 1 можно перенести на случай произвольного $\beta \in(0,1]$ : для любого элемента $f \in A_{1}(D)$ верна оценка

$$
\left\|f-G_{m}(f)\right\| \leqslant C|f|_{A_{1}(D)} m^{-\gamma(\beta) /(2(2+\gamma(\beta)))}
$$

где

$$
\gamma(\beta)=\min \left\{x>1:(1+x)^{1 /(2+x)}\left(1+\frac{1}{1+x}\right)=1+\frac{2-\beta}{x}\right\} .
$$

Заметим, что функция $\gamma(\beta)$ убьвает на $[0,1]$. При этом

$$
\frac{\gamma(0)}{2(2+\gamma(0))}=0.305 \ldots
$$

Сравнение этого результата с [3] показывает, что при малых $\beta$ скорость сходимости жадного алгоритма, вообще говоря, быстрее, чем при $\beta=1$.

В заключение хочу поблагодарить С.В.Конягина и В. Ю. Протасова за полезные обсуждения проблемы и многочисленные ценные замечания.

\section{СПИСОК ЦИТИРОВАННОЙ ЛИТЕРАТУРЫ}

1. DeVore R. A., Temlyakov V. N. // Adv. Comput. Math. 1996. V. 5. P. 173-187. 2. Konyagin S. V., Temlyakov V. N. // East J. Approx. 1999. V. 5. № 4. P. 493-499. 3. Livshitz E. D., Temlyakov V. N. // Constructive Approx. 2003. V. 19. № 4. P. 509-523. 4. Temlyakov V.N. Greedy expansions in Banach spaces // Research report 2003:06. Columbia, SC: Dept. Math. Univ. South Carolina, 2003. 